\title{
DSP-ALGORITHMS FOR CARDIAC SYMPTOMS INVESTIGATIONS
}

\author{
Ivan Kalchev \\ Faculty of Automatics, Technical University of Sofia, BG-1797, Sofia, Bulgaria, idk@tu-sofia.bg
}

\begin{abstract}
The achievements of the advanced medicine are not possible without the use of technical sciences. The aspiration for perfect instrumentation and for high precision of the technical systems for analysis of cardiac activity are still a challenge to engineers. The invention of more effective and with better quality indexes systems will permit a fast diagnostics, therefore a better medical treatment.

In the present paper the main cardiac-vessel symptoms are described and an possible algorithm for program assurance of $\mu$ P-system for cardiac investigations is proposed. The system is based on ADU 824 type of microprocessor, but the programs are compiled in MATLAB 4.2 environment.
\end{abstract}

Keywords: - cardiac symptoms, algorithms, signal processing

\section{BASIC TERMINOLOGY AND POSTULATION}

The ECG-signals are main data-carrier of pathological changes in the heart functioning. In fact, they are surface potentials descending from the electric waves' propagation through strongly defined structural parts, namely:

- SA - nodes

- AV - nodes

- Muscle of the auricles

- Auricles

- Left and right valves.

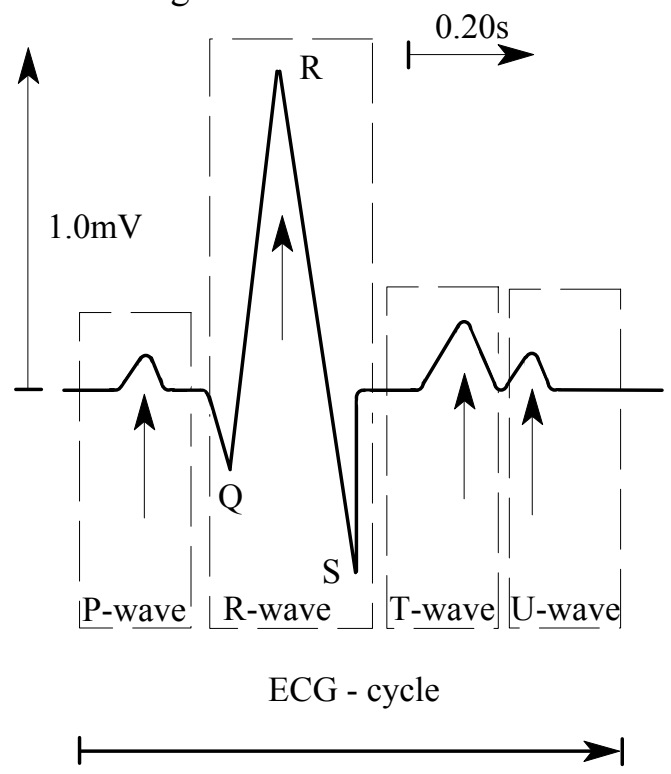

Fig.1 - Schematic drawing of a QRS-complex.
The most important characteristics, shown in Fig. 1. are as follows:

- P-wave

- T-wave

- QRS-wave,

but the base line of the signal is defined as a line between two neighboring P-Q intervals. Such a base line is an object of all the further amplitude measurements.

\section{PROBLEMS OF THE BASE LINE STABILITY}

The dispersion of the base line in the ECGrecords is a classical problem, for which there is not any universal solution yet.

This fact leads to invalid data while defining the main parameters. Usually, such a dispersion is around $0,5 \mathrm{~Hz}$ and less, but in the stress-test clinic cases the limit can be greater. The base line drift is suppressed by filtering.

In the present paper, as a result of the analysis of the classical methods for stabilization of the base line, it has been made the next inference. The use of some cascade adaptive filters for rejection of the decomponent, adaptive pulse filter for rejection of the rest dispersion unlinked with the QRS-complex, and FIR adaptive filter of second order for noise depressing, realized as a grate can yield fully passable results.

The implementation of the above mentioned adaptive FIR filters in medical electronic devices is 
due to the fact, that the transmitter characteristic of a typical DAQ-systems' channel is usually unstationary. It imposes a need of dynamic updating of the filters' coefficients in order to correspond better with the identification model.

There are known two modes of operation.

- back modeling, and

- forward modeling of the FIR - filters.

\section{DSP-ALGORITHMS FOR ECG- SIGNALS PROCESSING}

The main phases of the ECG-signals interpretation are, as follows:

- Calculating parameters

- Comparison of the results obtained with strongly defined requirements

- Formulation both of ECG conclusions, and diagnosis, and prescription for corresponding therapy.

The influence of the subjective factor during the visual interpretation can be avoided by use of advanced DSP-algorithms for morphologic, rate and screening analysis in the clinic practice. The software has to be open for additional functions (diagnostic and analysis). A method based on comparison of the absolute value of the differentiated ECG to a varying threshold value is proposed in [6]. Such a method is adaptive and selfsynchronized to the steep slope of the QRS, regardless of the amplitude and the sampling frequency of the record. In [7] are compared the noise sensitivity of nine algorithms, based on:

- amplitude and first derivative,

- first derivative only,

- first and second derivative, and

- digital filtering.

The most part of the problems with QRScomplex identification are decided by Daskalov and Christov [8] In the present paper algorithms for diagnostics of the main heart rate disturbances, arrhytmias and some more important program segments, performed in MATLAB 4.2 environment are given.

The informative parameters of the ECG-signals after a data compression are stored in the computer memory. In such a time an intrinsic part of the DAQ-system is the R-tooth detector.

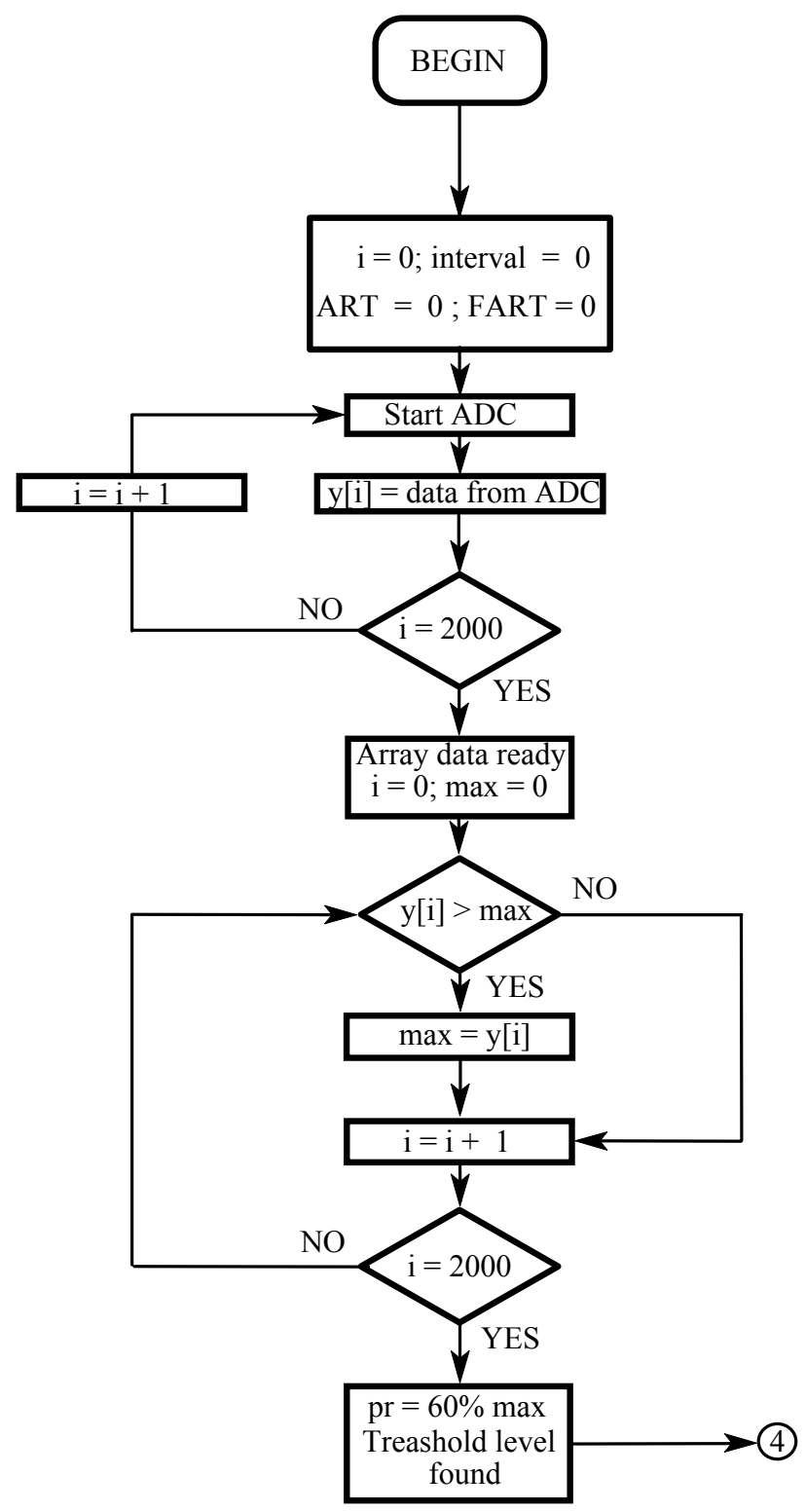

Fig.2a - Algorithm of the functioning.

In order to design an interactive algorithm it is of vital importance to assure a possibility for easy way criteria's changing. In such a case every cardiology could account the specific patient illness.

There are known the next rate disturbances:

- keen bradycardia (a critical rate drop),

- tachycardia (heavy increasing of the rate),

- asystolia and fibrillation (the non synchronized oscillations of the heart are called fibrilations),

- extrasystolic phenomena $(\mathrm{R}+\mathrm{T})$,

- bigamy or trigamy. 


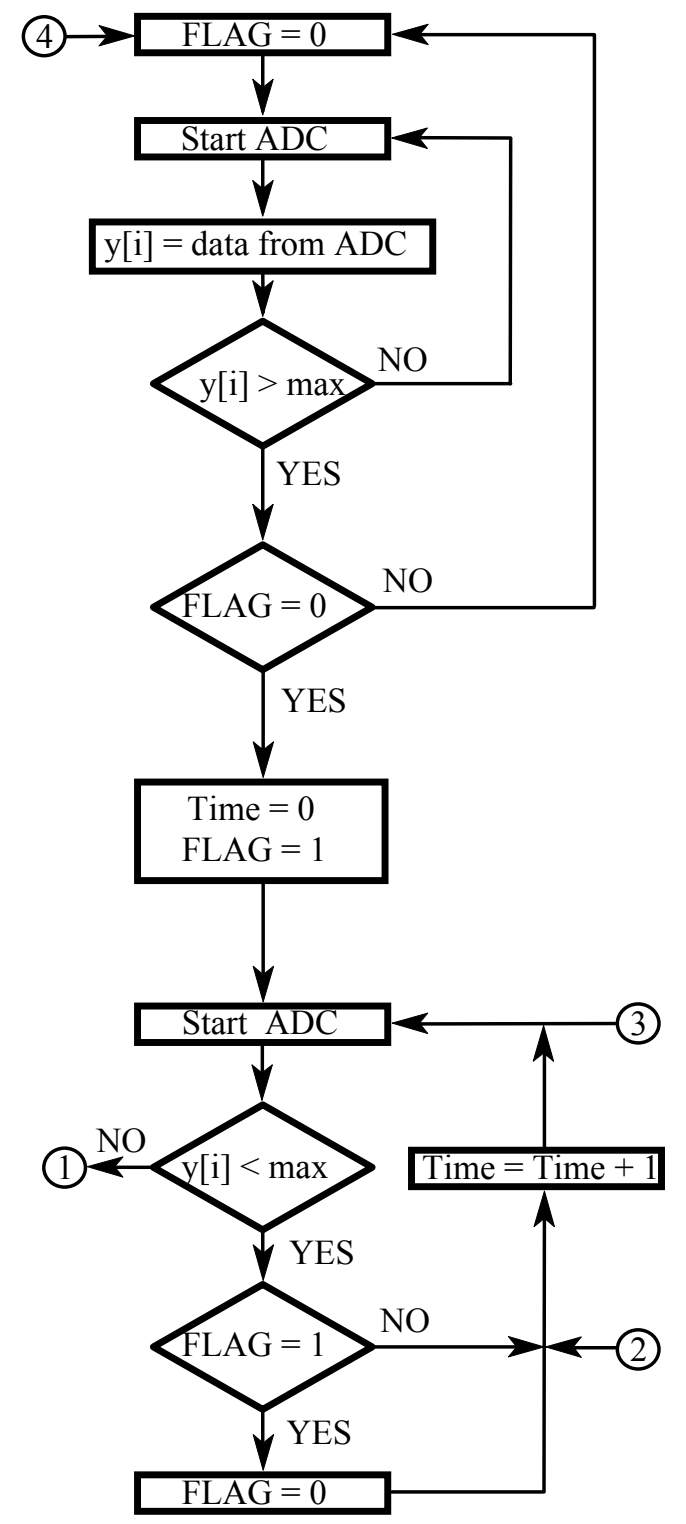

Fig.2b - Algorithm of the functioning.

The main algorithm of functioning for diagnostics of different rate disturbances is shown in Fig. 2.

\section{DISCUSSION, INFERENCES AND CONCLUSION}

The domain of ECG clinical investigations provides many opportunities for intelligent measuring instrumentation. A correct diagnosing the state of the heart in most cases is rely upon the availability of appropriate data base and knowledge. In such a time an effective clinical decision making involves the processing based on the objective criteria excluding subjective influencing factors.

Finally let see what the problem is. In author's opinion the program assurance provides major challenges for further development of microprocessor-based measurement systems.

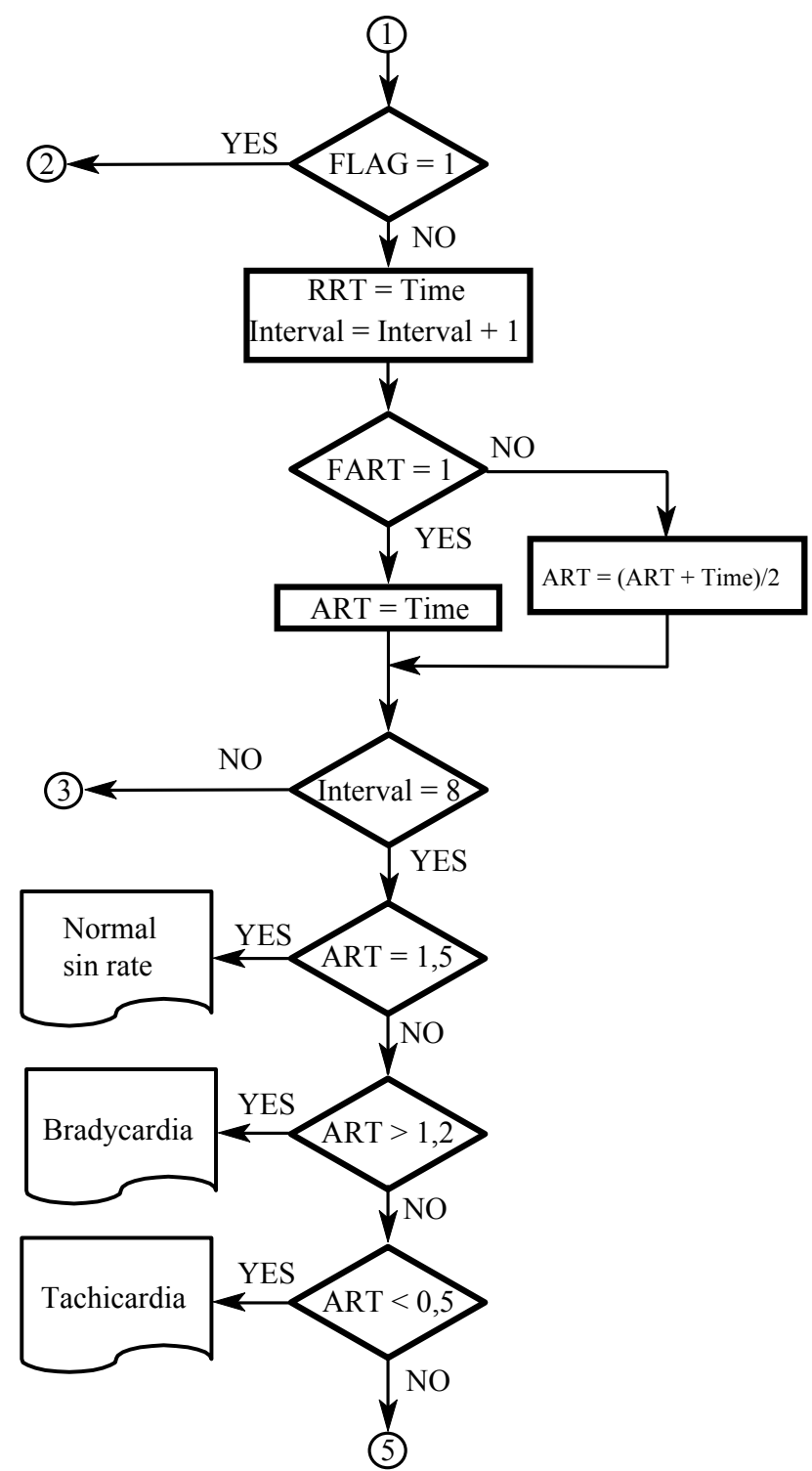

Fig.2c - Algorithm of the functioning.

A significant part of intelligent measurement systems used in practice needs advanced software products for signal interpretation in its clinical context.

Such measurements, in essence, are an typical example of the so called model-based measurements.

Model-based measurement is used when there are parameters which can not be measured directly, but which could yield important information if they could be estimated.

The diagnosis is the goal in the therapeutic practice and appropriately designed models can assist the clinician in their decision-making activity of patient state. Every human diagnostic process is simultaneously logical, associative, fuzzy and probabilistic that is why there are not possible strongly defined decision-making rules.

From what has been said above it can be done the next inferences:

- The design of medical instrumentation involve a special filtering and stabilization of the ECG-signals, 
- The lack of a clear formulation of some physiological processes influences on the choice of suitable measurement methods,

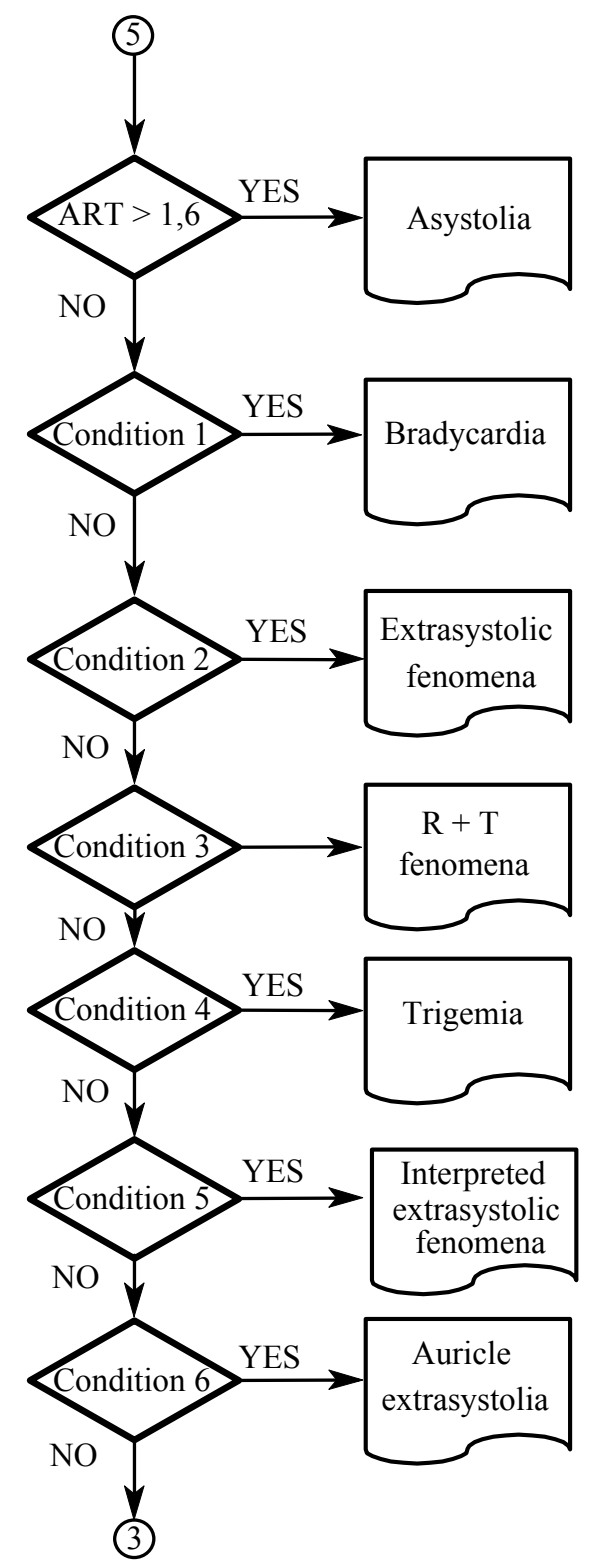

Condition 1: RRT > 1.9[ART(k-1)]

Condition 2: RRT[k-1] + RRT[k] = 2ART[k-2]

Condition 3: RRT[k-1] $<0.33$ ART[k-4]

Condition 4: RRT[k-2] $<0.9 A R T[k-3]$

Condition 5: RRT[k-1] + RRT $=$ ART[k-2]

Condition 6: RRT[k-1] =0.9ART[k-2]

Fig. 2d - Algorithm of the functioning.

- The program assurance of these intelligent systems is very complex due to the availability of numerous varying parameters.

\section{REFERENCES}

[1] Program MATLAB documentation: Wavelet user's guide.

[2] P. Laguna, N. Tharor, P. Caminal, R. Jane. Adaptive Base line Wander Removal in ECG. Ragnar Granit Institute, 1999.

[3] Perez D. Canceling $50 \mathrm{~Hz}$ Interferences in ECGs. Polytechnic University of Valencia, Spain, 1995.

[4] I. Stoyanov, N. Mudrov, I. Christov, I. Dotsinsky. ECG computer-based system, E+E No. 1-2/2202, pp.3-9.

[5] I. Dotsinsky, I. Christov, I. Daskalov. Twelvelead electrocardiogram obtained by eight leads, E+E No. 1-2/2202, pp.10-12.

[6] I. Christov, V. Stoyanov. Steep slope method for real time QRS detection, $E+E$ No. 1-2/2202, pp.13-17.

[7] G. Freisen. A comparison of the noise sensitivity of nine QRS detection algorithms, IEEE Trans. on Biomedical Engineering, No. 37, 1990, pp.85-98

[8] I. Daskalov, I. Christov. Improvement of resolution in measurement of electrocardiogram $R R$ intervals by interpolation, Medical Engineering \& Physics, No. 19, 1997, pp.375-379.

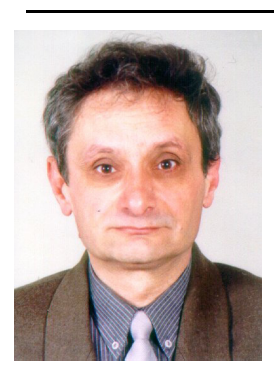

Ivan D. Kalchev (25 Oct. 1947), Bulgarian, graduated in computer science at the Kiev Politechnical Institute, Kiev, Ukraine (1971) and received the Ph.D. degree in measurement science from the Technical University of Sofia, Bulgaria (1991). He has published more than 100 research papers on $\mu P$ Based systems. Dr. Ivan Kalchev worked as associate researcher 10 years at the Central Institute for Computing Technique and Technologies - Sofia.

Became lecturer at the Department of Measurement, Technical University of Sofia (1993).

He teaches an university course of Electrical Measurements, and another of Distributed Measurement Systems. Since May, 1997 he is Associate Professor in measurement science at the $T U$ - Sofia (Bulgaria). In 2002 Mr. I. Kalchev received the $D$.Sc. degree in technical sciences from the TU-Sofia. Award: "Prof. ITALO GORINI MEMORIAL AWARD" - Torino, ITALY, 1994. Areas of special interest: measurement and distributed instruments; statistics and probabilities theory; digital signal processing. 